\title{
STUDY OF POLYMER ELASTIC BEHAVIOR IN THE DISPLACEMENT OF OIL DROPS AT PORE SCALE
}

\author{
LUIS PRADA, ARLEX CHAVES, JULIO PEDRAZA, JOSE GÓMEZ \& SAMUEL MUÑOZ \\ Universidad Industrial de Santander, Colombia
}

\begin{abstract}
Polymeric liquids have been used in the oil industry, especially at enhanced oil recovery (EOR). From the rheological point of view, polymers have the particularity of being viscoelastic liquids. One of the most common and useful models to describe that behavior is the upper convected Maxwell model (UCM). The main characteristic of the polymer used in the EOR process is the increase in viscosity which pushes the oil outside of the reservoir. The elasticity could contribute to the drag of the oil that stays in the reservoir. Studying the elastic effect on the oil drop at the pore scale, brings an explanation if the addition of elastic force could mobilize the oil. This research explores if the contraction and expansion of the polymer in the pore-scale may increase the elastic behavior of this kind of fluid. For that reason, this work simplified the pore geometry and built two simple geometries with micrometer lengths. Using source terms with the user defined function this work introduces the UCM model in the ANSYS Fluent simulator with the purpose of evaluating the elastic effect of the polymer in a contraction and expansion geometry. Also, using the Eulerian multiphase model this research considers the possibility that extra elastic force will show a deformation effect on the oil, for that reason, this work considers an oil drop on the upper wall of the geometry. Finally, all the simulations exhibit that at pore scale conditions extra vortices exist in the UCM model but it is not possible to deform the oil completely and push it outside of the restrictions.
\end{abstract}

Keywords: ANSYS Fluent, interfacial fluids mechanics, polymers, pore scale, viscoelasticity.

\section{INTRODUCTION}

Polymerflooding is an enhanced oil recovery (EOR) mechanism that improves the sweep efficiency of the water injected, to increase oil production. However, for decades have been considered that even with the viscosity of the polymer is not possible to decrease the oil trapped at the pore. Some research as is mention in this paper have been probing that the elastic behavior of the polymer is the main factor to consider in these phenomena.

Wreath [1] was the first scientist to consider the polymer effect at the reduction of the residual oil because of the viscoelastic potential. He compares the polymerflooding between two types of rocks, where at the more heterogeneity rock (Antolini) get a reduction of the residual oil. Wang et al. [2] studied many configurations of the residual oil trapped at the pore. He found that polymer could deform the oil volume that is trapped on the concavity of the rock or thinning the oil film that sticks on the wall of the pore. He explains that polymers are large and flexible entangled chains, therefore, when they move, they interact between each other creating elongation and movement of the other molecules, connecting with the oil that stay on the concavity. Researchers such as Urbissinova et al. [3], Seright [4] and Qi et al. [5] found in their experiments a reduction in at least $5 \%$ of the oil saturation with a polymerflooding process, increasing that value when they used more viscoelastic polymer.

For the simulation aspect, Yin et al. [6] use the upper convective Maxwell model to describe the viscoelastic behavior of the polymer solution. They show the contour of the stream function and the velocities for different Weissenberg and Reynold numbers in simplified pore geometries. With the increase of the elasticity of the polymer Yin et al. [6], found that the velocity of the polymer increases at the corner where the oil could be trapped, for that reason there is a chance for the polymer to decrease the oil saturation in that places. 
Afsharpoor and Balhoff [7] studied four pore geometries and evaluated the effects of the pressure, velocity and normal stress around the oil drop during the polymerflooding. This research indicated that the increase in elasticity could change the oil drop shape. Finally, Fan et al. [8] use the upper convective Maxwell (UCM) to evaluate the effect of high Weissenberg number in the polymerflooding at pore scale. Fan conclude that at higher Weissenberg number the normal stress increase, creating favorable condition to the deformation of the oil that stay on the reservoir. In this paper, the pore-scale flooding processes were investigated with the volume of fluid method for enhanced oil recovery. A single pore body/throat was established to investigate the wettability and interfacial tension, and viscoelastic effects on the polymer/oil flow interaction in pore scale. The results can provide some insights into the basic understanding of the polymer flooding process for enhanced oil recovery.

\section{MATHEMATICAL MODEL}

In this study, the computational fluid dynamic (CFD) technique was used, specifically the ANSYS Fluent software to evaluate the effect of the viscoelastic component of the polymer on the phenomena of the polymerflooding at the pore scale. The CFD modeling is applied here for viscoelastic flow around stationary oil droplets. First, as a problem in $2 \mathrm{~d}$ geometry governing equations: momentum and continuity can be written as follows:

$$
\begin{gathered}
\frac{\partial u}{\partial x}+\frac{\partial v}{\partial y}=0 \\
\rho\left(\frac{\partial u}{\partial t}+u \frac{\partial u}{\partial x}+v \frac{\partial u}{\partial y}\right)=-\frac{\partial p}{\partial x}+\frac{\partial \tau_{x x}}{\partial x}+\frac{\partial \tau_{x y}}{\partial y}, \rho\left(\frac{\partial v}{\partial t}+u \frac{\partial v}{\partial x}+v \frac{\partial v}{\partial y}\right)=-\frac{\partial p}{\partial y}+\frac{\partial \tau_{x y}}{\partial x}+\frac{\partial \tau_{y y}}{\partial y},
\end{gathered}
$$

where $u, v$ are the $x$ and $y$ component of the velocity, $\rho$ is density, $\tau_{x x}, \tau_{x y}$ and $\tau_{y y}$ are the stresses.

\subsection{Upper convected Maxwell model}

In this study the UCM model (eqn (3)) is used as the constitutive equation for the nonNewtonian fluid. The motivation of using this model is because it was developed to generalize the linear viscoelastic model to be valid under varying conditions of stress and motion, and suggested that describes the rheology of HPAM, one of the polymers most used in enhanced oil recovery. The UCM is given by:

$$
\boldsymbol{\tau}+\lambda \stackrel{\nabla}{\boldsymbol{\tau}}=2 \mu \boldsymbol{D},
$$

where $\boldsymbol{\tau}$ is the stress tensor, $\lambda$ is the relaxation time, $\boldsymbol{D}$ is the rate of deformation tensor, $\mu$ is the viscosity of the polymeric solution and $\boldsymbol{\tau}$ is the upper convected derivative of the stress tensor defined as:

$$
\stackrel{\nabla}{\boldsymbol{\tau}}=\frac{D \tau}{D t}-(\nabla v)^{T} \cdot \tau-\tau \cdot \nabla v
$$

Eqn (3) could be written in Einstein notation as:

$$
\frac{\partial \tau_{i j}}{\partial t}+V_{k} \frac{\partial \tau_{i j}}{x_{k}}=\boldsymbol{\tau}_{k j} \frac{\partial v_{i}}{\partial x_{k}}+\boldsymbol{\tau}_{i k} \frac{\partial v_{j}}{\partial x_{k}}+\frac{\mu_{p}}{\lambda} \gamma_{i j}-\frac{1}{\lambda} \boldsymbol{\tau}_{i j}
$$

where $\gamma_{i j}$ is the rate of deformation tensor. 


\subsubsection{Implementation in ANSYS Fluent software}

Eqn (5) represents the viscoelastic constitutive equation and is embedded into the Fluent code through UDF functionality. In UDF, the general user-defined scalar transport as follows:

$$
\frac{\partial(\rho \phi)}{\partial t}+\frac{\partial}{\partial x_{i}}\left(F_{i} \phi-\Gamma \frac{\partial \phi}{\partial x_{i}}\right)=S_{\phi}
$$

In eqn (6), there are four terms to be customized: transient term, convection term, diffusion term and source term, where $\phi$ is the scalar, corresponding to components of the stress tensor $\tau_{i j}$ in eqn (5). $\Gamma$ is the diffusion coefficient, and is set equal to zero in the present calculations. The transient term corresponds to the first term on the left side in eqn (5), the convection term is the second term on the left side; the remaining term in eqn (5) were grouped in the source term $S_{\phi}$ as shown in the following expression for each one the four polymer stress components

$$
S_{\tau_{i j}}=\tau_{k j} \frac{\partial v_{i}}{\partial x_{k}}+\tau_{i k} \frac{\partial v_{j}}{\partial x_{k}}+\frac{\mu_{p}}{\lambda} \gamma_{i j}-\frac{1}{\lambda} \tau_{i j}
$$

\subsubsection{Two phase model}

Finally, to model the two-phase fluid (water-oil and oil-polymer), the volume of fluid (VOF) model was used to model the two immiscible liquids by solving a single set of momentum equation and tracking the volume fraction of each of the fluids throughout the domain. The tracking of the interface(s) between the phases is accomplished by the solution of a continuity equation for the volume fraction of one (or more) of the phases. For the $q^{\text {th }}$ phase, this equation has the following form:

$$
\frac{1}{\rho_{q}}\left[\frac{\partial}{\partial t}\left(\alpha_{q} \rho_{q}\right)+\vec{\nabla} \cdot\left(\alpha_{q} \rho_{q} \overrightarrow{v_{q}}\right)=S_{\alpha_{q}}+\sum_{p=1}^{n}\left(\dot{m}_{p q}-\dot{m}_{q p}\right)\right]
$$

where $\dot{m}_{p q}$ is the mass transfer from phase $q$ to phase $p$ and $\dot{m}_{q p}$ is the mass transfer from phase $p$ to phase $q$. In the problem that we solve $S_{\alpha_{q}}$ is the source term we added before. The volume fraction equation will not be solved for the primary phase, the primary-phase will be computed based on the following constraint:

$$
\sum_{q=1}^{n} \alpha_{q}=1
$$

The fraction equation for this simulation was solver through implicit time discretization [9].

\section{VALIDATION}

Validation of the model is an important step in a simulation. In this paper, we present two kinds of validation: quantitative and qualitative validation.

\subsection{Quantitative validation}

The quantitative validation was performed comparing Yin et al. [6] velocities profiles to ANSYS Fluent simulation results, for the particular case of expansion geometry. Yin et al. used a relation of 4:1 height of the geometry and differences of Newtonian and nonNewtonian flows as Fig. 1 shows. For the case of Fig. 1(b), the Weissenberg number represent the ratio of a fluid time constant to a characteristic time of flow and is defined as a fluid time constant $\lambda$ multiplied with a deformation rate describing the flow. 


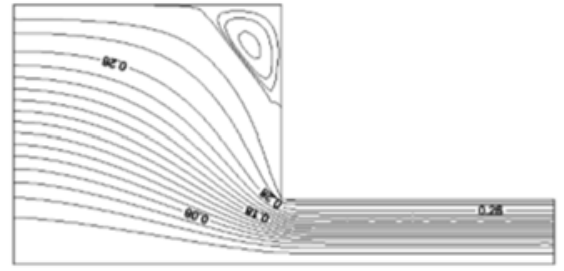

(a)

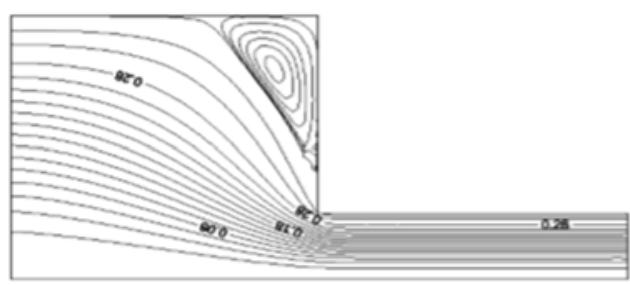

(b)

Figure 1: Streamlines in [6]. (a) $\mathrm{We}=0$; and (b) $\mathrm{We}=0.6$.

To validate Yin's results, a geometry of micrometer size was built because representing the pore scale, with the same 4:1 geometry relation. Properties of the mesh and other conditions are presented in Table 1. Using this condition for the geometry and fluid in ANSYS simulation, it is easy to observe that an increase in the viscoelasticity of the fluid creates more vorticity on the right corner of the geometry as Fig. 2 illustrated, that is in agreement with the results obtained by Yin.

Table 1: Properties of the fluid simulated.

\begin{tabular}{|l|c|}
\hline Properties & Value \\
\hline Flow velocity $(\mathrm{m} / \mathrm{s})$ & $1 * 10^{-5}$ \\
\hline Viscosity $(\mathrm{kg} / \mathrm{m}-\mathrm{s})$ & 0.0001 \\
\hline Density $\left(\mathrm{kg} / \mathrm{m}^{3}\right)$ & 1000 \\
\hline We & 0 and 6 \\
\hline Reynold & $1 * 10^{-5}$ \\
\hline
\end{tabular}

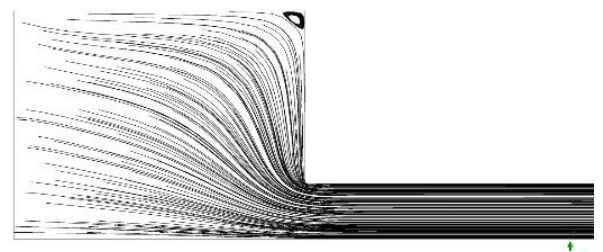

(a)

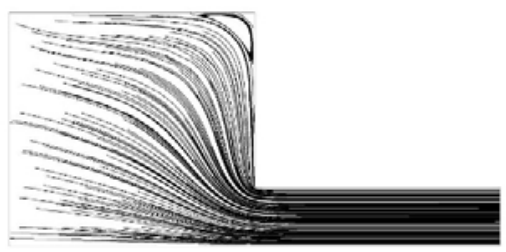

(b)

Figure 2: Streamlines in ANSYS Fluent. (a) $\mathrm{We}=0$; and (b) $\mathrm{We}=0.6$.

\subsection{Qualitative validation}

The qualitative validation was performed comparing results of velocities for the Rayleigh problem of a viscoelastic fluid. The Rayleigh problem consider a fluid initially at rest $(t=0)$ on an infinitely long flat plate. For $t>0$, the plate moves in the right direction, and the liquid is putting in motion as shown in Fig. 3. 


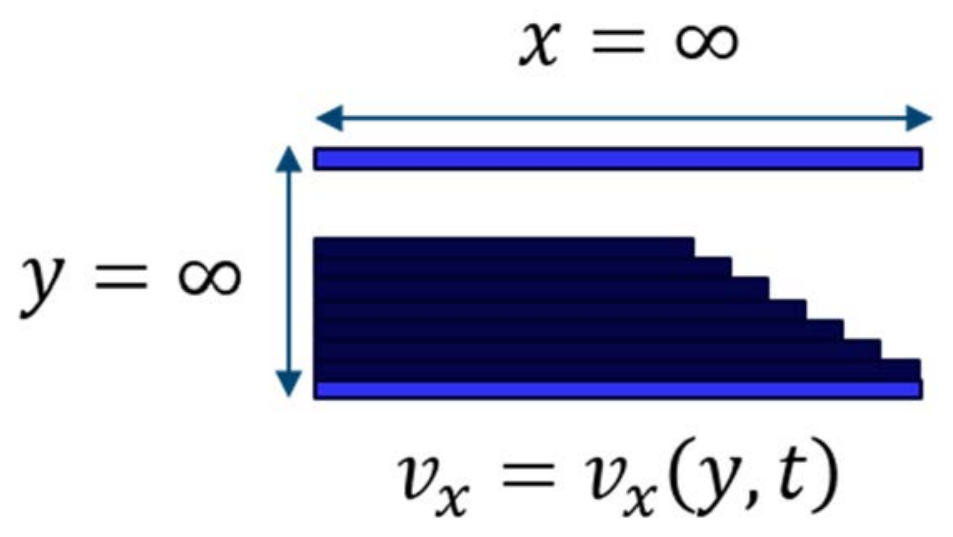

Figure 3: Rayleigh-type problem.

The analytical solution for the Rayleigh-type problem for a non-Newtonian fluid was developed by Fetecau and Fetecau [10] using the Upper Convective Maxwell model and is given by the following expression:

$$
\begin{gathered}
v(y, t)=V-\frac{2 V}{\pi} \int_{0}^{1 /(2 \sqrt{v} \lambda)} \frac{r_{3} e^{\left(r_{4} t\right)}-r_{4} e^{\left(r_{3} t\right)}}{r_{3}-r_{4}} \times \frac{\sin (y \xi)}{\xi} d \xi-\frac{2 V}{\pi} e^{\left(-\frac{t}{2 \lambda}\right)} \int_{1 /(2 \sqrt{v} \lambda)}^{\infty}\left[\cos \left(\frac{\gamma t}{2 \lambda}\right)+\right. \\
\left.\frac{1}{\gamma} \sin \left(\frac{\gamma t}{2 \lambda}\right)\right] \frac{\sin (y \xi)}{\xi} d \xi
\end{gathered}
$$

where

$$
r_{3,4}=\frac{-1 \pm \sqrt{1-4 v \lambda \xi^{2}}}{2 \lambda} \text { and } \gamma=\sqrt{4 v \lambda \xi^{2}-1}
$$

The values of velocity as a function of time and position were obtained from eqn (10) using the Wolfram Mathematica software, and then they was compared obtained from the simulation. Results of the comparison are illustrated in Figs 4-6.

As Figs 4-6 show, the simulations in ANSYS for a UCM model is very similar to the analytical solution in [10], that demonstrate that is possible to continue with the next topic of the research, the evaluation of the polymer with the oil at different geometries.

\section{RESULTS}

In this study, the simulations of the oil-water, oil-polymer and oil-polymer UDF systems were compared. The purposes of the simulation were analyzed if at the end of the transient simulation the oil trapped on the corner of expansion or contraction geometry could move to the outlet.

Two of the most important characteristics in an oil reservoir are the wettability and the surface tension between the fluid. The wettability is the preferences to adhere to the rock a drop of oil or other fluid. For that reason, this paper present different values of surface tension $(0.01,0.005$ and $0.001 \mathrm{n} / \mathrm{m})$ and wettability $(120,90$ and 60 grades $)$ for the same systems to analyze the effect of this characteristic in the oil displacement. The properties of the fluid in the simulation are in Table 2. 


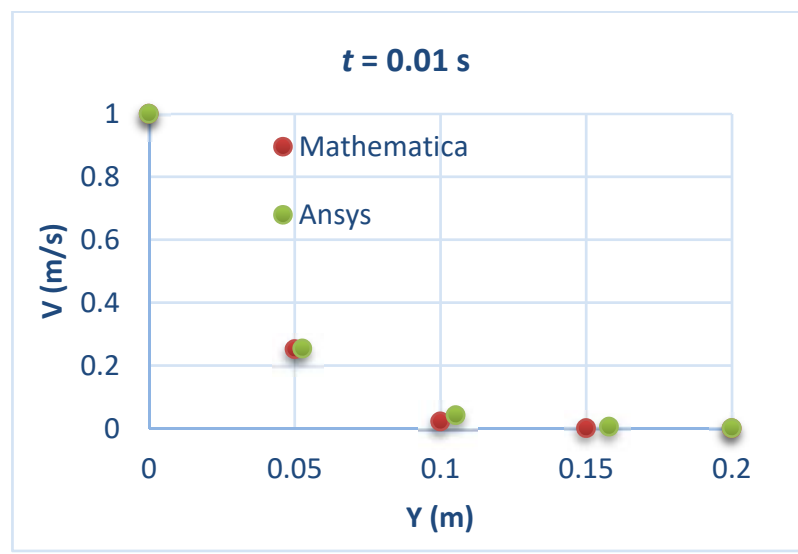

Figure 4: Velocities at different position at $t=0.01 \mathrm{~s}$.

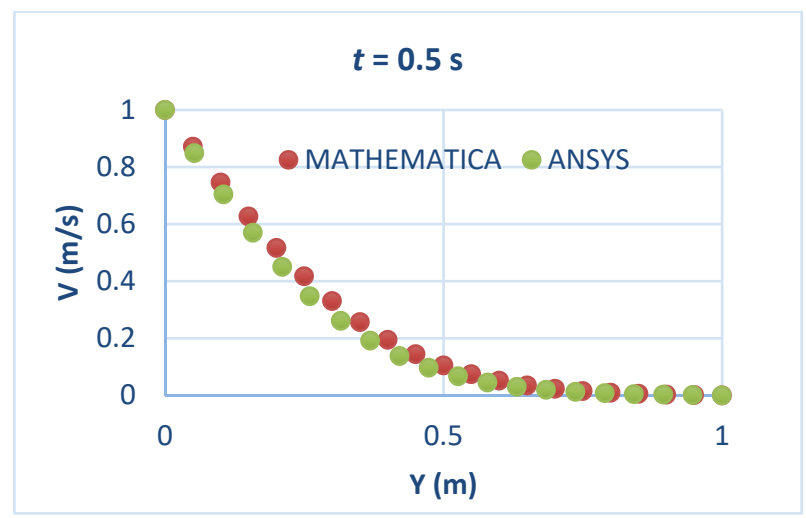

Figure 5: Velocities at different position at $t=0.5 \mathrm{~s}$.

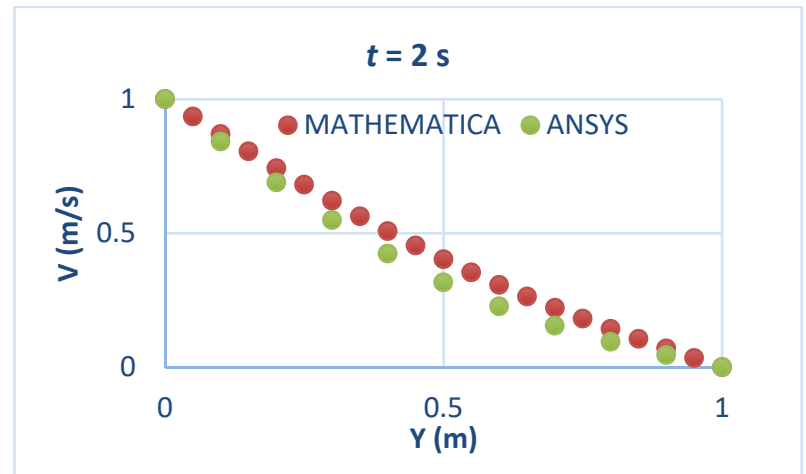

Figure 6: Velocities at different position at $t=2 \mathrm{~s}$. 
Table 2: Properties of the fluids simulated.

\begin{tabular}{|l|c|}
\hline Properties of oil & Value \\
\hline Viscosity $(\mathrm{kg} / \mathrm{m}-\mathrm{s})$ & 0.1 \\
\hline Density $\left(\mathrm{kg} / \mathrm{m}^{3}\right)$ & 900 \\
\hline Properties of water & Value \\
\hline Viscosity $(\mathrm{kg} / \mathrm{m}-\mathrm{s})$ & 0.001 \\
\hline Density $\left(\mathrm{kg} / \mathrm{m}^{3}\right)$ & 998.2 \\
\hline Properties of polymer & Value \\
\hline Viscosity $(\mathrm{kg} / \mathrm{m}-\mathrm{s})$ & 0.01 \\
\hline Density $\left(\mathrm{kg} / \mathrm{m}^{3}\right)$ & 900 \\
\hline
\end{tabular}

In this research we consider two simple geometries: contraction and expansion. Additionally, a small part of the volume is an oil drop (red region), the rest of the volume is the other fluid (blue region) that could be water or polymer, as Fig. 7 shows.

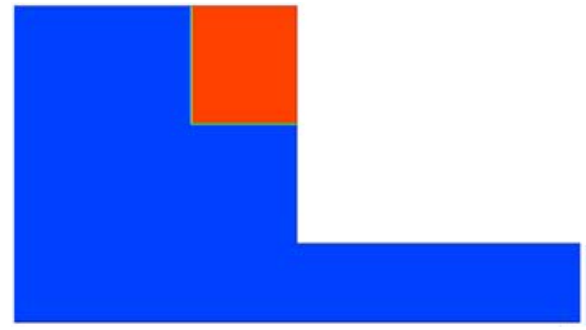

(a)

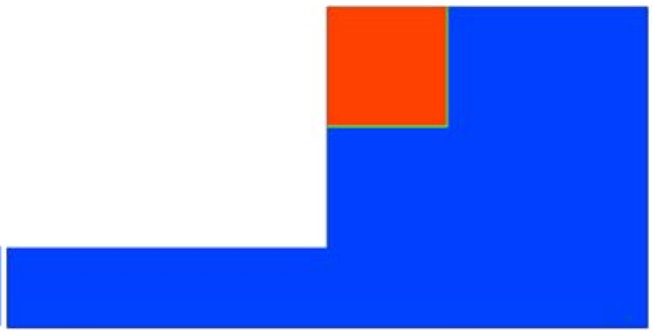

(b)

Figure 7: Oil drop at pore channel. (a) Contraction; and (b) Expansion.

\subsection{Contraction geometry}

For the contraction geometry, Fig. 8 shows that the most critical parameter that affects the oil movement is the wettability, represented in the contact angle. In the angle of 120 grades, in each one of the three values of surface tension evaluated ${ }_{2}$ all the drop of oil moved out of the geometry, however for the other two angles, the oil drop is divided but not total evacuated.

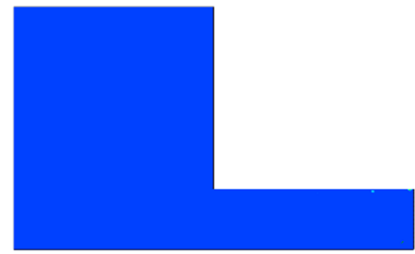

(a)

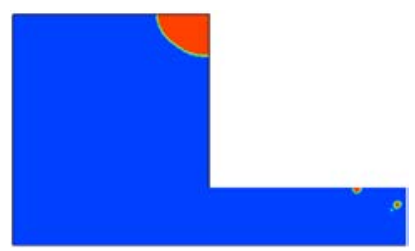

(b)

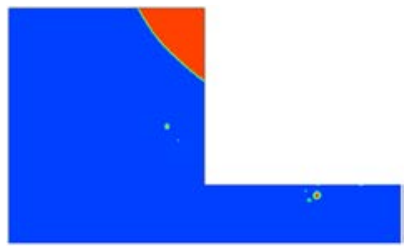

(c)

Figure 8: Oil drop at different angles of wettability. (a) 120 grades; (b) 90 grades; and (c) 60 grades. 
Fig. 9 shows results for the interfacial tension aspect, at least for the values of $0.01,0.005$ and $0.001 \mathrm{n} / \mathrm{m}$ does not represent change on the oil drop in comparison of the effect of the contact angle, because for these three values the volume of the oil drop that is removed remains the same as in the previous case.

Additionally, the polymer with UDF source only represents a little movement in the oil drop, as Fig. 10 illustrates. The polymer with the viscoelastic behavior moves more oil than the polymer without these characteristics in the cases of 90 and 60 grades. For these two simulations, even the oil drops look smaller in comparison with the simulation without the viscoelastic model.

\subsection{Expansion geometry}

For the expansion geometry, investigating streamlines between viscoelastic fluids and Newtonian fluids, there is an increase in the vortex of the corner as Fig. 11 shows. That behaviour probably will increase the oil that stay on the corner of the geometry.

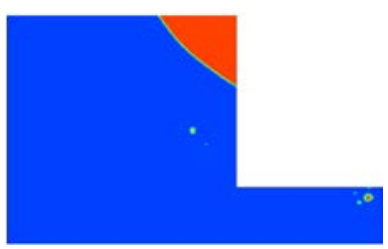

(a)

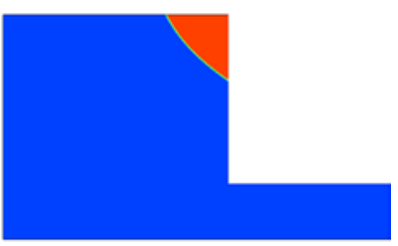

(b)

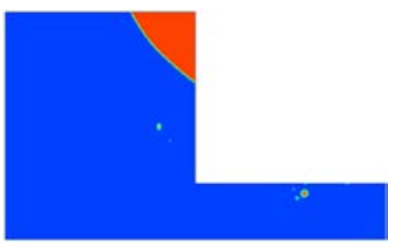

(c)

Figure 9: Oil drop at different interfacial tension. (a) $0.01 \mathrm{n} / \mathrm{m}$; (b) $0.005 \mathrm{n} / \mathrm{m}$; and (c) $0.001 \mathrm{n} / \mathrm{m}$.

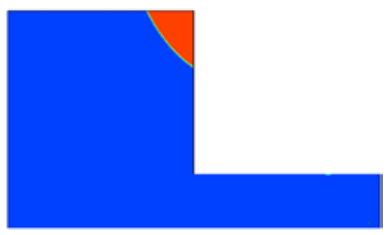

(a)

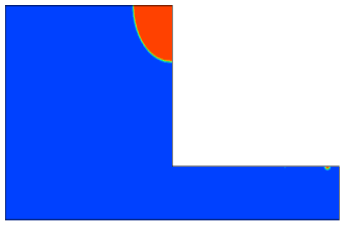

(b)

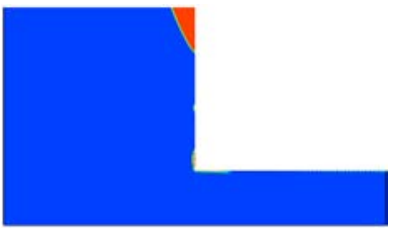

(c)

Figure 10: Oil drop at different systems. (a) Oil-water; (b) Oil-polymer; and (c) Oilpolymer UDF.

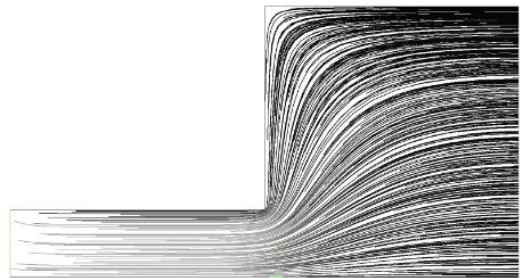

(a)

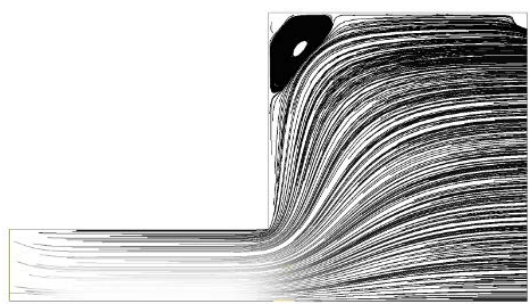

(b)

Figure 11: Streamlines in ANSYS Fluent. (a) Newtonian fluid; and (b) Viscoelastic fluid. 
In the case of two fluids, all the simulations have the same oil trapped on the corner, even with the changes of the system, interfacial tension, and wettability the oil drop stay on the corner. Fig. 12 shows the different shapes of the drop, but it was not possible at least in the conditions of this work to move out the oil of the geometry. The main reason is that the fluid could arrive at that corner but not with enough forces to drag the oil drop.

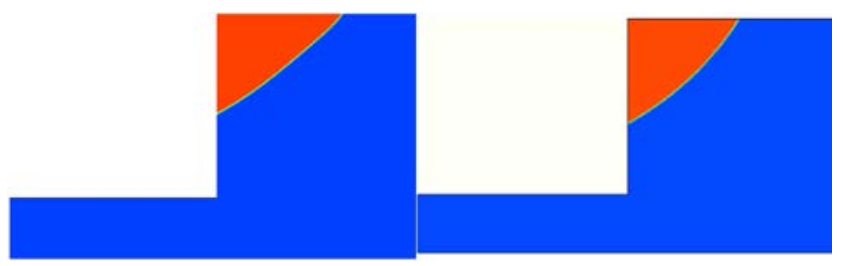

(a) (b)

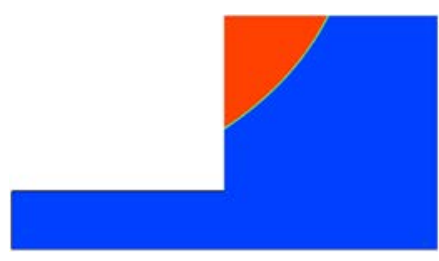

(c)

Figure 12: Oil drop at different systems at the same wettability (60 grades) and interfacial tension $(0.001 \mathrm{n} / \mathrm{m})$. (a) Oil-water; (b) Oil-polymer; and (c) Oil-polymer UDF.

\section{ACKNOWLEDGEMENTS}

This work was supported by Universidad Industrial de Santander, and the research groups: FIRST (Fenomenos Interfaciasles, Reologia y Simulacion de Transporte) and GRM (Grupo de Recobro Mejorado).

\section{REFERENCES}

[1] Wreath, D.G., A study of polymerflooding and residual oil saturation. Dissertation, 1989.

[2] Wang, D. et al., Viscous-elastic fluids can mobilize oil remaining after water-flood by force parallel to the oil-water interface. SPE Asia Pacific Improved Oil Recovery Conference, Society of Petroleum Engineers, 2001.

[3] Urbissinova, T., Trivedi, J.J. \& Kuru, E., Effect of elasticity during viscoelastic polymer flooding: A possible mechanism of increasing the sweep efficiency. SPE Western Regional Meeting, Society of Petroleum Engineers, 2010.

[4] Seright, R.S., Use of Polymers to Recover Viscous Oil from Unconventional Reservoirs. Final Report, Contract No. DE-NT0006555, US Department of Energy, 2011.

[5] Qi, P. et al., Reduction of residual oil saturation in sandstone cores by use of viscoelastic polymers. SPE Journal, 22(2), pp. 447-458, 2017.

[6] Yin, H., Wang, D. \& Zhong, H., Study on flow behaviours of viscoelastic polymer solution in micropore with dead end. SPE Annual Technical Conference and Exhibition, Society of Petroleum Engineers, 2006.

[7] Afsharpoor, A. \& Balhoff, M., Static and dynamic CFD modelling of viscoelastic polymer: Trapped oil displacement and deformation at the pore-level. SPE Annual Technical Conference and Exhibition, Society of Petroleum Engineers, 2013.

[8] Fan, J.W. et al., Hydrodynamics of residual oil droplet displaced by polymer solution in micro-channels of lipophilic rocks. International Journal of Heat and Technology, 35(1), pp. 611-618, 2017.

[9] Ansys Inc., ANSYS Fluent UDF Manual, 2013.

[10] Fetecau, C. \& Fetecau, C., The first problem of Stokes for an Oldroyd-B fluid. International Journal of Non-Linear Mechanics, 38(10), pp. 1539-1544, 2003. 